\title{
PIECEWISE LINEAR BUNDLES IN THE METASTABLE RANGE
}

\author{
BY
}

\author{
KENNETH C. MILLETT
}

\begin{abstract}
For numerable vector bundles a nonzero section determines a unique trivial line subbundle containing the section and this subbundle is a direct summand of the bundle. The main result, a consequence of concordanceisotopy theory, states that in the metastable range a nonzero section to a piecewise linear $\mathbf{R}^{n}$ bundle determines a unique trivial line subbundle and that this is the best possible result. This fact is then compared with the known failure of the summand property below the stable range.
\end{abstract}

1. Introduction. One knows that the theories of linear and piecewise linear bundles are quite similar in the stable range [10] and that below this range various types of anomalous behaviour occur [7], [23]. Morlet [21] and others have thought that these pathologies might not occur in the metastable range. Recent results of Hatcher [11] and Volodin [26], however, have shown that this need not be the case, cf. [25]. Several consequences of this observation are discussed in light of the main purpose of this paper, a study of the consequences of nonzero sections in the metastable range. The main theorem states that, in this range, a nonzero section determines a unique trivial line bundle containing the section. We shall also show that this is the best possible result.

The main ingredients in the analysis of these questions are the results contained in my paper on piecewise linear concordances and isotopies [18]. Indeed the genesis of that research was the desire to study the unstable geometry of piecewise linear $\mathbf{R}^{n}$ bundles. The work of Kuiper and Lashof [17] provided the impetus for this study and serves as a basic reference.

Before beginning I wish to thank T. Akiba, P. Henry, R. Lashof and R. Stern for their comments and suggestions which were of great assistance during the course of this research.

2. Definitions and basic results. All of the definitions and results concern either the simplicial, block, or piecewise linear categories. For the definitions and

Received by the editors April 7, 1975.

AMS (MOS) subject classifications (1970). Primary 55F10, 55F25, 55F60, 57C50, 55G40; Secondary 55C35, 58P10.

Key words and phrases. Nonzero sections, piecewise linear bundles, trivial subbundles, space of piecewise linear embeddings, concordances, isotopies. 
properties of the simplicial category see Curtis [9], for the block category see Rourke and Sanderson [22], and for the piecewise linear category see either Zeeman [27] or Hudson [14].

Objects in the simplicial and block categories are distinguished by a tilde $(\sim)$ appearing over the objects in the block category. Thus $\Pi_{i}(X)$ and $\Pi_{i}(\widetilde{X})$ denote the $i$ th homotopy groups of an object in the simplicial category and the $i$ th block-homotopy groups of a related object in the block category. It will be important to compare these groups by including them in an exact sequence, cf. Morlet [21],

$$
\rightarrow \Pi_{i}(X) \rightarrow \Pi_{i}(\widetilde{X}) \rightarrow \Pi_{i}^{\mathrm{rel}}(X) \rightarrow
$$

This is accomplished by defining $\Pi_{i}^{\text {rel }}(X)$ to be the group of (simplicial) arc components of $\Omega^{i} \widetilde{X}$ which do not contain elements of $\Omega^{i} X$, where the "loops" are taken in the appropriate category.

Let $M$ and $N$ denote compact piecewise linear manifolds of dimensions $m$ and $n$ and boundaries $\partial M$ and $\partial N$, respectively. Let $\Delta^{s}$ denote the standard $s$ dimensional simplex, $I=[0,1], r J=[-r, r],(r J)^{n}=r D^{n}$, and $\Sigma^{n-1}=\partial D^{n}$. A subspace $\left(K, K_{0}\right)$ of a space $\left(L, L_{0}\right)$ is said to be proper if $K \backslash K_{0} \subset L \backslash L_{0}$. A piecewise linear map $f:\left(K, K_{0}\right) \rightarrow\left(L, L_{0}\right)$ is proper if $\left(f(K), f\left(K_{0}\right)\right)$ is a proper subcomplex of $\left(L, L_{0}\right)$.

Definition (2.1). Given a proper piecewise linear embedding of a proper subcomplex of $(M, \partial M)$ in $(N, \partial N), f:\left(K, K_{0}\right) \rightarrow(N, \partial N)$, let $E(M, N ; f)$ denote the simplicial complex of locally unknotted proper piecewise linear embeddings of $(M, \partial M)$ into $(N, \partial N)$ extending $f$. That is the simplicial complex whose $s$ simplices are proper piecewise linear embeddings

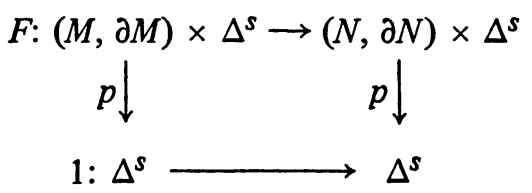

such that

(i) the diagram is commutative;

(ii) $F^{-1}\left(\partial N \times \Delta^{s}\right)=\partial M \times \Delta^{s}$;

(iii) $F \mid\left(K, K_{0}\right) \times \Delta^{s}=f \times 1$;

(iv) for any simplex $\Delta$ linearly embedded in $\Delta^{s},(N \times \Delta, F(M \times \Delta))$ is a locally unknotted manifold pair.

If $f$ is not specified this complex is denoted by $E(M, N)$.

The block complexes, $\widetilde{E}(M, N ; f)$ and $\widetilde{E}(M, N)$, are defined as above except that condition (i) is replaced by

(i) for all faces $\Delta^{\prime}$ of $\Delta^{s}, F \mid(M, \partial M) \times \Delta^{\prime}$ is an embedding of $(M, \partial M) \times$ $\Delta^{\prime}$ into $(N, \partial N) \times \Delta^{\prime}$. 
Definition (2.2). Let $\left(K, K_{0}\right)$ be a piecewise linear space. The complexes of proper piecewise linear homeomorphisms of $\left(K, K_{0}\right)$ onto itself which are fixed on $K_{0}$ are denoted $H\left(K, K_{0}\right)$ and $\tilde{H}\left(K, K_{0}\right)$. If $K$ is a manifold the subcomplex of $H\left(K, K_{0}\right)$ of homeomorphisms which are fixed on $\partial K \cup K_{0}$ is denoted by $H_{\partial}\left(K, K_{0}\right)$. If $K_{0}$ is empty these spaces are denoted by $H(K)$ and $H_{\partial}(K)$, respectively.

REMARK (2.3). Inasmuch as all the definitions have analogues in the block category which are apparent, given those in the simplicial category, only the simplicial definitions will be given.

Definition (2.4). The complexes of germs of proper embeddings of $(M, \partial M)$ into $(N, \partial N)$ extending $f$ and of germs of homeomorphisms of $K$ which extend $f$ are denoted by $G E(M, N ; f)$ and $\operatorname{GH}\left(K ; K_{0}\right)$, respectively. The first, for example, consists of the simplices of the complexes of proper embeddings of neighborhoods $\left(U, U_{0}\right)$ of $\left(K, K_{0}\right)$ into $(N, \partial N)$ which extend $f \mid K_{0}$.

Definition (2.5). The complex of proper piecewise linear maps of $\left(L, L_{0}\right)$ into $\left(L^{\prime}, L_{0}^{\prime}\right)$ extending $f:\left(K, K_{0}\right) \rightarrow\left(L^{\prime}, L_{0}^{\prime}\right)$ is denoted by $M\left(\left(L, L_{0}\right),\left(L^{\prime}, L_{0}^{\prime}\right) ; f\right)$. If $\left(L, L_{0}\right)=\left(L^{\prime}, L_{0}^{\prime}\right)$ this complex is denoted by $M\left(L, L_{0} ; f\right)$.

Definition (2.6). Let $i:\left(L_{0}, L_{0}\right) \rightarrow\left(L, L_{0}\right)$ denote the inclusion. The subcomplex of $M\left(L, L_{0} ; i\right)$ consisting of homotopy equivalences is denoted by $N\left(L, L_{0}\right)$, that is to say if $F$ is an s-simplex of $N\left(L, L_{0}\right)$ there is another s-simplex $G$ of $N\left(L, L_{0}\right)$ such that $F G$ and $G F$ are homotopic to the identity through homotopies which are fixed on $L_{0}$, leave $L \backslash L_{0}$ setwise invariant, and respect the projection to $\Delta^{s} \times I$. If $L$ is a manifold and $L_{0}=\partial L$ then $N\left(L, L_{0}\right)$ is denoted by $N_{\partial}(L)$. The subcomplex of those equivalences keeping a subcomplex $K$ fixed is denoted by $N_{\partial}(L)_{K}$.

REMARK (2.7). It is easy to see that $\Pi_{j}\left(N\left(L, L_{0}\right)\right)$ is isomorphic to $\Pi_{j}\left(M\left(L, L_{0} ; i\right)\right)$, via the inclusion, for $j \geqslant 1$.

DEFINITION (2.8). The complex of proper concordances of (germs of) proper embeddings extending a proper embedding $f:\left(K, K_{0}\right) \rightarrow(N, \partial N)$ of a proper subcomplex of $(M, \partial M)$ is denoted by $C(M, N ; f)(G C(M, N ; f))$. It is the subcomplex of $E(I \times M, I \times N ; 1 \times f)(G E(I \times M, I \times N ; 1 \times f))$ whose s-simplices $F$ satisfy

(i) $F^{-1}(\{0\} \times N)=\{0\} \times M$, and

(ii) $F^{-1}(\{1\} \times N)=\{1\} \times M$.

DEFINITION (2.9). The complex of (germs of) proper isotopies of proper embeddings extending a proper embedding $f:\left(K, K_{0}\right) \rightarrow(N, \partial N)$ of a proper subcomplex of $(M, \partial M)$ is denoted by $I(M, N ; f)(G I(M, N ; f))$. It is the complex of paths in $E(M, N ; f)(G E(M, N ; f))$ or, equivalently, the subcomplex of $E(I \times M, I \times N ; 1 \times f)(G E(I \times M, I \times N ; 1 \times f))$ such that, for any s-simplex 
$F$, the diagram

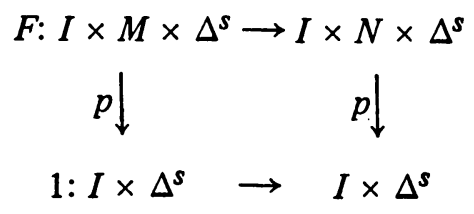

is commutative.

Definition (2.10). Let $f:(M, \partial M) \rightarrow(N, \partial N)$ be a proper piecewise linear embedding. $C_{\partial}(M, N ; f \mid K)\left(C_{\partial_{0}}(M, N ; f \mid K)\right)$ and $I_{\partial}(M, N ; f \mid K)\left(I_{\partial_{0}}(M, N ; f \mid K)\right)$ are the subcomplexes of $C(M, N ; f \mid \partial M \cup K)$ and $I(M, N ; f \mid \partial M \cup K)$, respectively, whose simplices $F$ satisfy the property that $F \mid\left(\{0,1\} \times M \times \Delta^{s}\right)=1 \times$ $f \times 1\left(F \mid\left(\{0\} \times M \times \Delta^{s}\right)=1 \times f \times 1\right)$. The analogous definitions for concordances and isotopies of germs of embeddings are also assumed.

Several simplicial fibrations and quasi-fibrations will be required in the next section. These are recognized via standard methods, cf. [17], [21], by employing Hudson's $s$ covering isotopy theorem.

Definition (2.11). Let $p: E \rightarrow B$ be a simplicial map and $G$ a simplicial group acting on $E$. Then $p: E \rightarrow B$ is a (weak) fiber bundle, with group $G$, if

(i) $p$ is onto (the arc components of $B$ which contain the image),

(ii) $G$ acts freely and transitively on $p^{-1}(b)$ for all $b \in B$ ( $b$ in the arc components of $B$ which contain the image).

Definition (2.12). Let $p: E \rightarrow B$ be a simplicial map and $F=p^{-1}\left(b_{0}\right)$. Then $p: E \rightarrow B$ is a (weak) quasi-fibration if $p_{*}: \Pi_{s}(E, F) \rightarrow \Pi_{s}\left(B, b_{0}\right)$ is an isomorphism for all $s$ (for all $s>0$ and 1-1 for $s=0$ ).

(2.13) Note that Kuiper and Lashof define $\operatorname{PL}(n, k)=G H\left(R^{n}, R^{k}\right)$ while here $P L(n, k)=H\left(R^{n}, R^{k}\right)$. As noted below these two groups have the same homotopy type. Let $\overline{P L}(n, k)=H_{\partial}\left(D^{n}, D^{k}\right)$.

To study these, and related spaces, it is necessary to consider several fibrations whose definitions are related. To begin, let $\gamma_{5}: H_{\partial}\left(D^{m} \times R^{n}, D^{m} \times R^{k}\right) \rightarrow$ $G H_{\partial}\left(D^{m} \times R^{n}, D^{m} \times R^{k}\right)$ be the projection of a homeomorphism to its germ. Next define

$$
\gamma_{1}: H_{\partial}\left(D^{m} \times D^{n}, D^{m} \times D^{k}\right) \rightarrow H_{\partial}\left(D^{m} \times R^{n}, D^{m} \times R^{k}\right),
$$

following Kuiper and Lashof [17, II] by taking a piecewise linear homeomorphism, $1 \times \rho_{1}$, of $D^{m} \times R^{n}$ to $D^{m} \times \operatorname{int}\left(1 / 2 D^{n}\right)$, followed by the element of $H_{\partial}\left(D^{m} \times D^{n}, D^{m} \times D^{k}\right)$, followed by inclusion into $D^{m} \times R^{n}$, and followed by an extension of the piecewise linear version of Kister's theorem to push the image onto $D^{m} \times R^{n}$. Let $\gamma_{3}=\gamma_{5} \circ \gamma_{1}$.

Similarly one can define

$$
\gamma_{2}: H_{\partial}\left(D^{m} \times \Sigma^{n}, D^{m} \times \Sigma^{k}\right) \rightarrow H_{\partial}\left(D^{m} \times R^{n}, D^{m^{\prime}} \times R^{k}\right)
$$


by taking a piecewise linear homeomorphism, $1 \times \rho_{2}$, of $D^{m} \times R^{n}$ to $D^{m} \times$ $\operatorname{int}\left(\Sigma_{+}^{n}\right)$, followed by, etc. Let $\gamma_{4}=\gamma_{5}^{\circ} \gamma_{2}$.

If ev denotes the appropriate evaluation and $N(K)$ denotes a variable neighborhood of $K$ one has the following useful proposition.

Proposition (2.14).

$$
H_{\partial}\left(D^{m} \times D^{n}, N\left(D^{m} \times\{0\}\right) \cup\left(D^{m} \times D^{k}\right)\right)
$$

$$
\begin{aligned}
& \longrightarrow H_{\partial}\left(D^{m} \times D^{n}, D^{m} \times D^{k}\right) \\
& \stackrel{\gamma_{3}}{\longrightarrow} G H_{\partial}\left(D^{m} \times R^{n}, D^{m} \times R^{k}\right)
\end{aligned}
$$

is $a$ (weak) fiber bundle if $m>0 \quad(m \geqslant 0)$;

$$
\begin{aligned}
H_{\partial}\left(D^{m} \times \Sigma^{n}, N\left(D^{m} \times(1,0, \ldots, 0)\right) \cup\left(D^{m} \times \Sigma^{k}\right)\right) \\
\longrightarrow H_{\partial}\left(D^{m} \times \Sigma^{n}, D^{m} \times \Sigma^{k}\right) \\
\stackrel{\gamma_{4}}{\longrightarrow} G H_{\partial}\left(D^{m} \times R^{n}, D^{m} \times R^{k}\right)
\end{aligned}
$$

is $a$ (weak) fiber bundle if $k \geqslant 0$ and $m>0 \quad(m \geqslant 0)$;

$$
H_{\partial}\left(D^{m} \times R^{n}, N\left(D^{m} \times\{0\}\right) \cup\left(D^{m} \times R^{k}\right)\right)
$$

$$
\begin{aligned}
& \longrightarrow H_{\partial}\left(D^{m} \times R^{n}, D^{m} \times R^{k}\right) \\
& \stackrel{\gamma_{5}}{\longrightarrow} G H_{\partial}\left(D^{m} \times R^{n}, D^{m} \times R^{k}\right)
\end{aligned}
$$

is a fiber bundle;

$$
H_{\partial}\left(D^{m} \times D^{n}, D^{m} \times\{0\}\right) \rightarrow H_{\partial}\left(D^{m} \times D^{n}\right) \stackrel{\mathrm{ev}}{\longrightarrow} E\left(D^{m}, D^{m} \times D^{n} ; j\right)
$$

is $a$ (weak) fiber bundle if $n \geqslant 3 \quad(n \geqslant 0)$.

$$
\begin{aligned}
H_{\partial}\left(D^{m} \times \Sigma^{n}, D^{m}\right. & \times(1,0, \ldots, 0)) \\
& \rightarrow H_{\partial}\left(D^{m} \times \Sigma^{n}\right) \stackrel{\mathrm{ev}}{\longrightarrow} E\left(D^{m}, D^{m} \times \Sigma^{n} ; j\right) .
\end{aligned}
$$

(2.20) Proof of Proposition (2.14). The proofs of each of the cases follows the same format. First in each case it is clear that the indicated fiber is a group which acts transitively and freely on each fiber. Thus it need only be shown that the projection is onto (or onto the arc components of the base which meet the image in the case of a weak fibration). The first three cases follow from the techniques of Microbundles and bundles. I [17] and Hudson's s-isotopy extension theorem [13] as indicated in the proof of Lemma 1.1 in Kuiper and Lashof. The final two cases are handled in much the same fashion in that Zeeman's un- 
knotting theorem [27] is employed in (2.18) with the s-isotopy extension theorem, while in (2.19) no statement concerning the image is given so that the $s$-isotopy extension theorem is sufficient.

Proposition (2.21). The following are contractible:

$$
\begin{aligned}
& H_{\partial}\left(D^{m} \times D^{n}, D^{m} \times D^{k}\right), H_{\partial}\left(D^{m} \times \Sigma^{n}, D^{m} \times\left(\Sigma_{+}^{n} \cup \Sigma^{k}\right)\right), \\
& H_{\partial}\left(D^{m} \times \Sigma^{n}, N\left(D^{m} \times(1,0, \ldots, 0)\right) \cup\left(D^{m} \times \Sigma^{k}\right)\right),
\end{aligned}
$$

and $H_{\partial}\left(D^{m} \times R^{n}, N\left(D^{m} \times\{0\}\right) \cup\left(D^{m} \times R^{k}\right)\right)$.

(2.22) The proof of Proposition (2.21) is a straightforward generalization of various forms of the Alexander isotopy [3] adapted to the piecewise linear category. For proofs, in a slightly less general case, the reader is referred again to Kuiper and Lashof. Their techniques are easily extended to prove (2.21).

Corollary (2.23). (i) $\Omega G H_{\partial}\left(D^{m} \times R^{n}, D^{m} \times R^{k}\right)$ is homotopy equivalent to $H_{\partial}\left(D^{m} \times D^{n}, N\left(D^{m} \times\{0\}\right) \cup\left(D^{m} \times D^{k}\right)\right)$, via $\lambda$,

(ii) $H_{\partial}\left(D^{m} \times \Sigma^{n}, D^{m} \times \Sigma^{k}\right)$ is homotopy equivalent to $\mathrm{GH}_{\partial}\left(D^{m} \times R^{k}, D^{n} \times R^{k}\right)$, via $\gamma_{4}$ modulo arc components,

(iii) $H_{\partial}\left(D^{m} \times R^{n}, D^{m} \times R^{k}\right)$ is homotopy equivalent to $G_{\partial}\left(D^{m} \times R^{n}, D^{m} \times R^{k}\right)$, via $\gamma_{5}$,

(iv) $E\left(D^{m}, D^{m} \times D^{n} ; j\right)$ is contractible if $n \geqslant 3$.

This corollary follows easily from Propositions (2.14) and (2.21), and standard facts for simplicial fibrations.

CoRollary (2.24). If $f:\left(D^{m}, \Sigma^{m-1}\right) \rightarrow\left(D^{m+n}, \Sigma^{m+n-1}\right)$ is a proper piecewise linear embedding and $n \geqslant 3$, then $E\left(D^{m}, D^{m+n} ; f\right)$ is contractible.

(2.25) Proof of (2.24). Zeeman's unknotting theorem [27] implies that there is a proper piecewise linear homeomorphism $F: D^{m+n} \rightarrow D^{m+n}$ extending $f$, i.e., $F \mid D^{m} \times\{0\}=f . F$ induces a homeomorphism $F^{\prime}: E\left(D^{m}, D^{m} \times D^{n} ; j\right) \rightarrow$ $E\left(D^{m}, D^{m+n} ; f \mid \Sigma^{m-1}\right)$ and thereby proves the corollary.

An extension of the proof of Lemma (1.7) of Kuiper and Lashof [17] gives the following proposition which is required for the description of several useful quasi-fibrations.

Proposition (2.26). The following inclusions are homotopy equivalences:

(i)

$$
H_{\partial}\left(D^{m+1} \times \Sigma^{n-1}, D^{m+1} \times \Sigma^{k-1}\right)
$$

$$
\stackrel{i_{1}}{\longrightarrow} H_{\partial}\left(D^{m} \times D^{n}, N\left(D^{m} \times\{0\}\right) \cup\left(D^{m} \times D^{k}\right)\right),
$$


(ii)

$$
H_{\partial}\left(D^{m} \times \Sigma^{n}, D^{m} \times\left(\Sigma_{+}^{n} \cup \Sigma^{k}\right)\right)
$$

$$
\stackrel{i_{2}}{\longrightarrow} H_{\partial}\left(D^{m} \times \Sigma^{n}, N\left(D^{m} \times(1,0, \ldots, 0)\right) \cup\left(D^{m} \times \Sigma^{k}\right)\right),
$$

where $i_{1}$ takes $D^{m+1} \times \Sigma^{n-1}$ to $D^{m} \times\left(D^{n} \backslash\right.$ int $\left.1 / 2 D^{n}\right)$ and extends by the identity and $i_{2}$ is the usual inclusion.

PROPOSITION (2.27). The following are quasi-fibrations:

$$
H_{\partial}\left(D^{m+1} \times \Sigma^{n-1}, D^{m+1} \times \Sigma^{k-1}\right) \longrightarrow H_{\partial}\left(D^{m} \times D^{n}, D^{m} \times D^{k}\right)
$$

$$
\begin{aligned}
H_{\partial}\left(D^{m} \times \Sigma^{n}, D^{m} \times\left(\Sigma_{+}^{n} \cup \Sigma^{k}\right)\right) & \stackrel{\gamma_{1}}{\longrightarrow} H_{\partial}\left(D^{m} \times R^{n}, D^{m} \times R^{k}\right), \\
\stackrel{\gamma_{2}}{\longrightarrow} H_{\partial}\left(D^{m} \times \Sigma^{n}, D^{m} \times \Sigma^{k}\right) & \left.R^{m} \times R^{k}\right)
\end{aligned}
$$

where $j: D^{m} \rightarrow D^{m} \times \Sigma^{n}$ is defined by $j(x)=(x,(1,0, \ldots, 0))$ and $\gamma_{1}$ and $\gamma_{2}$ are defined below.

(2.30) Quasi-fibrations (2.28) and (2.29). Let 1 denote the germ of the identity homeomorphism as well as the identity homeomorphism. Since the fiber $\gamma_{5}$ is contractible

$$
\gamma_{5^{*}}: \Pi_{s}\left(H_{\partial}\left(D^{m} \times R^{n}, D^{m} \times R^{k}\right),\{1\}\right) \rightarrow \Pi_{s}\left(G H_{\partial}\left(D^{m} \times R^{n}, D^{m} \times R^{k}\right),\{1\}\right)
$$

is an isomorphism. Consider the diagrams:

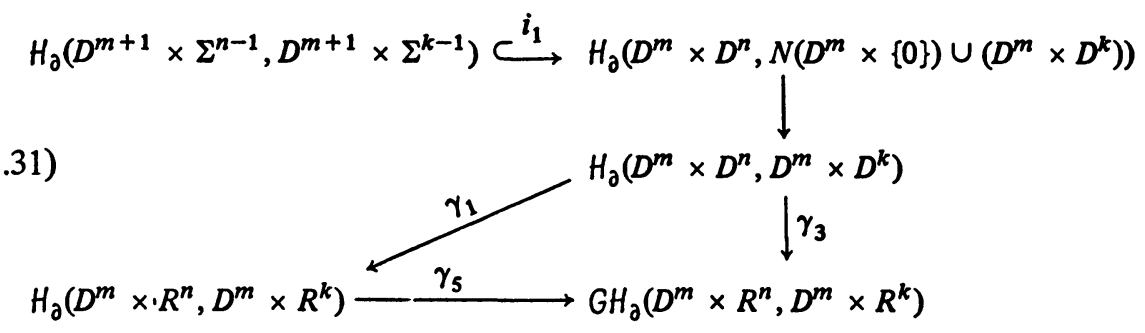

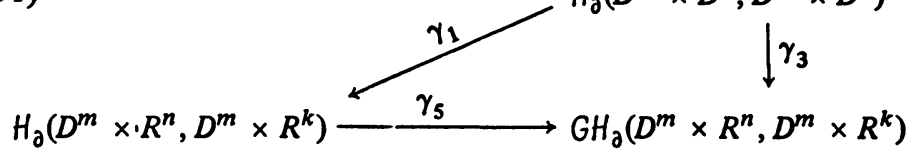

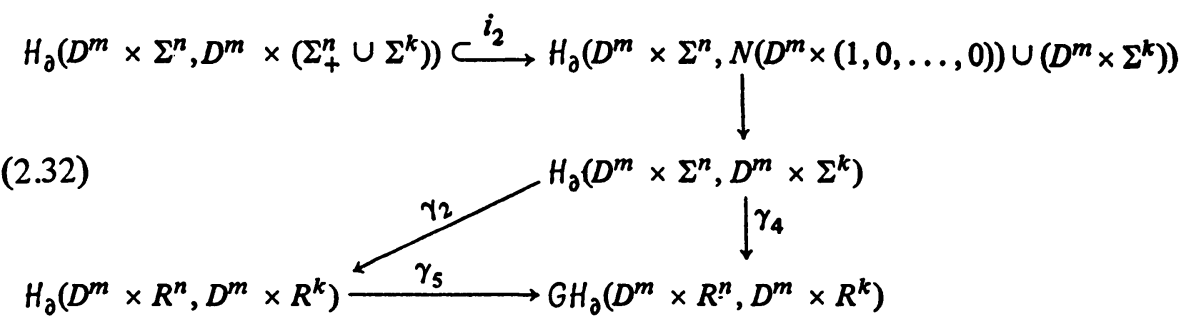


Clearly,

$$
\begin{aligned}
& \gamma_{1}^{-1}(1)=i_{1}\left(H_{\partial}\left(D^{m+1} \times \Sigma^{n-1}, D^{m+1} \times \Sigma^{k-1}\right)\right) \text { and } \\
& \gamma_{2}^{-1}(1)=i_{2}\left(H_{\partial}\left(D^{m+1} \times \Sigma^{n-1}, D^{m+1} \times \Sigma^{k-1}\right)\right) .
\end{aligned}
$$

Therefore

$$
\begin{gathered}
\Pi_{s}\left(H_{\partial}\left(D^{m} \times D^{n}, D^{m} \times D^{k}\right), i_{1}\left(H_{\partial}\left(D^{m+1} \times \Sigma^{n-1}, D^{m+1} \times \Sigma^{k-1}\right)\right)\right) \\
\gamma_{1 *} \downarrow \\
\Pi_{s}\left(H_{\partial}\left(D^{m} \times R^{n}, D^{m} \times R^{k}\right),\{1\}\right)
\end{gathered}
$$

and

$$
\begin{gathered}
\Pi_{s}\left(H_{\partial}\left(D^{m} \times \Sigma^{n}, D^{m} \times \Sigma^{k}\right), i_{2}\left(H_{\partial}\left(D^{m} \times \Sigma^{n}, D^{m} \times\left(\Sigma_{+}^{n} \cup \Sigma^{k}\right)\right)\right)\right) \\
\gamma_{2^{*}} \downarrow \\
\Pi_{s}\left(H_{\partial}\left(D^{m} \times R^{n}, D^{m} \times R^{k}\right),\{1\}\right)
\end{gathered}
$$

are isomorphisms since $\gamma_{3}=\gamma_{5} \circ \gamma_{1}$ and $\gamma_{4}=\gamma_{5} \circ \gamma_{2}$. Thus (2.28) and (2.29) are quasi-fibrations if $m>0$. If $m=0$ then $\gamma_{3}$ and $\gamma_{4}$ fail to be onto, they miss the orientation reversing component, and therefore give only a weak quasi-fibration.

Proposition (2.33). As far as homotopy groups are concerned, there is an exact sequence for a "fibration"

$$
H_{\partial}\left(D^{m} \times \Sigma^{n}, D^{m} \times(1,0, \ldots, 0)\right) \rightarrow \Omega H_{\partial}\left(D^{m-1} \times R^{n+1}, D^{m-1} \times\{0\}\right)
$$

$$
\rightarrow E\left(D^{m}, D^{m} \times \Sigma^{n} ; j\right) .
$$

(2.35) Proof. Let $k=0$. By Corollary (2.20) and Proposition (2.26) there is a homotopy equivalence " $\left(i_{1}^{-1}\right)$ " $\circ \lambda \circ\left(\Omega \gamma_{5}\right)=\alpha$ from $\Omega H_{\partial}\left(D^{m-1} \times R^{n+1}, D^{m-1} \times\{0\}\right)$ to $H_{\partial}\left(D^{m} \times \Sigma^{n}\right)$. Also there is a germ map, analogous to $\gamma_{4}$, giving the fibration

$$
\begin{aligned}
& H_{\partial}\left(D^{m} \times \Sigma^{n}, N\left(D^{m} \times(1,0, \ldots, 0)\right)\right) \downarrow \\
& H_{\partial}\left(D^{m} \times \Sigma^{n}, D^{m} \times(1,0, \ldots, 0)\right) \\
& \downarrow^{\gamma_{6}} \\
& G H_{\partial}\left(D^{m} \times R^{n}, D^{m} \times\{0\}\right)
\end{aligned}
$$

which is a homotopy equivalence since its fiber is contractible, by (2.21). Thus “ $\left(\gamma_{6}^{-1}\right)$ " $\circ \gamma_{5}=\beta$ is a homotopy equivalence from $H_{\partial}\left(D^{m} \times R^{n}, D^{m} \times\{0\}\right)$ to 
$H_{\partial}\left(D^{m} \times \Sigma^{n}, D^{m} \times(1,0, \ldots, 0)\right)$. Since (2.19) is a fiber bundle, $m>0$, and $\alpha$ and $\beta$ are homotopy equivalences there is an exact sequence for

$$
\begin{gathered}
H_{\partial}\left(D^{m} \times R^{n}, D^{m} \times\{0\}\right) \stackrel{\text { “ }\left(\alpha^{-1}\right) " \circ \beta}{\longrightarrow} \Omega H_{\partial}\left(D^{m-1} \times R^{n+1}, D^{m-1} \times\{0\}\right) \\
\stackrel{(\mathrm{ev}) \circ \alpha}{\longrightarrow} E\left(D^{m}, D^{m} \times \Sigma^{n} ; j\right) .
\end{gathered}
$$

A fundamental result of the study of concordances and isotopies [18] is the following theorem which provides the key to the metastable theory of nonzero sections.

THEOREM (2.37). Let $f:\left(D^{m}, \Sigma^{m-1}\right) \rightarrow(N, \partial N)$ be a proper piecewise linear embedding. If $\Pi_{j}(N)=0, j \leqslant k$ and $n-m \geqslant 3$, then $\Pi_{s}\left(C_{\partial}\left(D^{m}, N\right), I_{\partial}\left(D^{m}, N\right)\right)=0$ for $s \leqslant n+k-m-3$.

3. The metastable theory of nonzero sections. In employing the methods developed in the previous section to the study of nonzero sections to piecewise linear bundles a key piece of information is supplied by the following theorem. Let $G_{n+1}$ denote $h\left(\Sigma^{n}\right)$ and $F_{n}$ denote $h\left(\Sigma^{n},(1,0, \ldots, 0)\right)$. Note that suspension gives an inclusion of $G_{n}$ as a subcomplex of $F_{n}$.

Proposition (3.1). If $n \geqslant 3$ there is a homomorphism

$$
\Pi_{s}\left(E\left(D^{m}, D^{m} \times \Sigma^{n} ; j\right)\right) \rightarrow \Pi_{s+m}\left(\Sigma^{n}\right) \oplus \Pi_{s+m}\left(F_{n}, G_{n}\right),
$$

where $j: D^{m} \rightarrow D^{m} \times \Sigma^{n}$ is given by $j(x)=(x,(1,0, \ldots, 0))$, which is an isomorphism if $s<2 n-3$ and an epimorphism if $s=2 n-3$.

(3.2) Proof of Proposition (3.1). The proof is by induction on $s$. The case $s=0$ is precisely Corollary 2.18, page 454, of Rourke and Sanderson [22] which states, in effect, that $\Pi_{0}\left(E\left(D^{m}, D^{m} \times \Sigma^{n} ; j\right)\right)$ is isomorphic to $\Pi_{m}\left(G_{n+1}, G_{n}\right)$ for $n>2$. It is easily noted that $\Pi_{m}\left(G_{n+1}, G_{n}\right) \cong \Pi_{m}\left(\Sigma^{n}\right) \oplus$ $\Pi_{m}\left(F_{n}, G_{n}\right)$ and that the homomorphism from $\Pi_{0}\left(E\left(D^{m}, D^{m} ; j\right)\right)$ onto the $\Pi_{m}\left(\Sigma^{n}\right)$ summand is defined by taking the homotopy class of the projection of the embedding, relative to its boundary, in $\Sigma^{n}$.

Assume, inductively, that the proposition has been verified for $s=0$, $1, \ldots, k-1$. First $\Pi_{k}\left(E\left(D^{m}, D^{m} \times \Sigma^{n} ; j\right)\right) \cong \Pi_{k-1}\left(\Omega E\left(D^{m}, D^{m} \times \Sigma^{n} ; j\right)\right)$. Since $\Omega E\left(D^{m}, D^{m} \times \Sigma^{n} ; j\right)=I_{\partial}\left(D^{m}, D^{m} \times \Sigma^{n}\right), \Pi_{k-1}\left(\Omega E\left(D^{m}, D^{m} \times \Sigma^{n} ; j\right)\right) \cong$ $\Pi_{k-1}\left(I_{\partial}\left(D^{m}, D^{m} \times \Sigma^{n}\right)\right)$. By Theorem (2.37), if $n \geqslant 3$ and $k<2 n-3 \quad(k=$ $2 n-3)$, the inclusion of $I_{\partial}\left(D^{m}, D^{m} \times \Sigma^{n}\right)$ in $C_{\partial}\left(D^{m}, D^{m} \times \Sigma^{n}\right)$ induces an isomorphism (epimorphism) on $\Pi_{k-1}$. Furthermore $C_{\partial}\left(D^{m}, D^{m} \times \Sigma^{n}\right)$ is $E\left(D^{m+1}, D^{m+1} \times \Sigma^{n} ; j\right)$ so that by induction $\Pi_{k-1}\left(C_{\partial}\left(D^{m}, D^{m} \times \Sigma^{n}\right)\right)$ is isomorphic to $\Pi_{k+m}\left(\Sigma^{n}\right) \oplus \Pi_{k+m}\left(F_{n}, G_{n}\right)$. The composition of these isomorphisms gives the isomorphism 


$$
\Pi_{k}\left(E\left(D^{m}, D^{m} \times \Sigma^{n} ; j\right)\right) \cong \Pi_{k+m}\left(\Sigma^{n}\right) \oplus \Pi_{k+m}\left(F_{n}, G_{n}\right) .
$$

One also notes that the projection of $\Pi_{k}\left(E\left(D^{m}, D^{m} \times \Sigma^{n} ; j\right)\right)$ onto the $\Pi_{k+m}\left(\Sigma^{n}\right)$ summand is defined by taking the homotopy class of the projection of the $k$ simplex $F$ of embeddings, relative to the total boundary, $p(2) \circ F: D^{m} \times \Delta^{k} \rightarrow \Sigma^{n}$.

Let $1 P L(n)=H\left(R^{n},\{0,(1,0, \ldots, 0)\}\right)$ and $P L(n, k)=H\left(R^{n}, R^{k}\right)$ denote the simplicial groups of piecewise linear $R^{n}$ bundles with nowhere zero sections and trivial sub- $R^{k}$-bundles, respectively.

Proposition (3.3). If $n \geqslant 4$ there is a homomorphism

$$
\Pi_{s}(1 P L(n), P L(n, 1)) \rightarrow \Pi_{s}\left(F_{n-1}, G_{n-1}\right),
$$

which is an isomorphism if $s<2 n-4$ and an epimorphism if $s=2 n-4$.

Corollary (3.4). A nowhere zero section to a PL(n) bundle, $n \geqslant 4$, over a complex $K$ lies in a (unique) subline bundle if $\operatorname{dim}(K)<2 n-3(\operatorname{dim}(K)<$ $2 n-4)$.

Above this range this need not be the case as $\Pi_{s}\left(F_{n-1}, G_{n-1}\right)$ may be nonzero. The following corollary of (3.3) contradicts a result of T. Akiba [1], [2].

COROLLARY (3.5). There is a PL(n) bundle, $n \geqslant 4$, over a sphere having a nowhere zero section which does not lie in a unique subline bundle.

Another result of these fibrations and Proposition (3.2) takes a somewhat different, but equivalent, form. Let the quotient space $\operatorname{PL}(n+k) / P L(n+k, k)$ denote the piecewise linear Stiefel manifold $V_{n+k, k}^{P L}$ of [32].

COROLlaRY (3.6). For $n \geqslant 4$ there is a homomorphism

$$
\Pi_{s}\left(V_{n, 1}^{P L}\right) \rightarrow \Pi_{s}\left(G_{n}, G_{n-1}\right),
$$

which is an isomorphism if $s<2 n \leqslant 4$ and an epimorphism if $s=2 n-4$.

(3.7) Proof of Proposition (3.3) ANd Corollaries (3.4), (3.5) AND (3.6). Taking $m=0$ and $k=0,1$ in (2.28) there are homotopy equivalences of $\Omega P L(n)=\Omega H\left(R^{n},\{0\}\right)$ and $\Omega P L(n, 1)=\Omega H\left(R^{n}, R^{1}\right)$ with $H_{\partial}\left(D^{1} \times \Sigma^{n-1}\right)$ and $H_{\partial}\left(D^{1} \times \Sigma^{n-1}, D^{1} \times \Sigma^{0}\right)$, respectively. By $(2.36)$ " $\left(\gamma_{6}^{-1}\right) " \cdot \gamma_{4}$ gives a homotopy equivalence of $H_{\partial}\left(D^{1} \times \Sigma^{n-1}, D^{1} \times \Sigma^{0}\right)$ to

$$
H_{\partial}\left(D^{1} \times \sum^{n-1}, D^{1} \times\{(1,0, \ldots, 0)\}\right.
$$

thereby giving the sequence

$$
\Omega P L(n, 1) \rightarrow \Omega P L(n) \rightarrow C\left(D^{1}, D^{1} \times \Sigma^{n-1} ; j\right) .
$$

On the other hand one has the standard fibration

$$
1 P L(n) \rightarrow P L(n) \rightarrow E\left((1,0, \ldots, 0), R^{n} \backslash\{0\}\right)
$$

where the base space is homotopically $\Sigma^{n-1}$, cf. Kuiper and Lashof. The loop of this fibration can be then compared with (3.8) by 


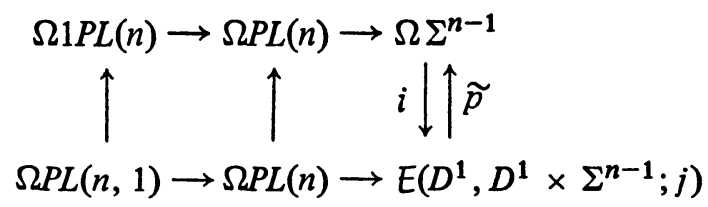

where $\tilde{p}$ is the projection given by $\tilde{p}(f)=p(2) \circ f$ and $i$ is the inclusion. The homotopy lattice of this diagram [19] gives an isomorphism

$$
\Pi_{s-1}\left(E\left(D^{1}, D^{1} \times \Sigma^{n-1} ; j\right), i\left(\Omega \Sigma^{n-1}\right)\right) \cong \Pi_{s-1}(\Omega 1 P L(n), \Omega P L(n, 1)) .
$$

The exact sequence of this pair of base complexes and Proposition (3.1) implies that there is a homomorphism

$$
\Pi_{s-1}\left(E\left(D^{1}, D^{1} \times \Sigma^{n-1} ; j\right), i\left(\Omega \Sigma^{n-1}\right)\right) \rightarrow \Pi_{s}\left(F_{n-1}, G_{n-1}\right)
$$

which is an isomorphism if $s<2 n-4$ and an epimorphism if $s=2 n-4$ proving the proposition.

A result of James [15] implies that $\Pi_{s}\left(F_{n-1}, G_{n-1}\right)=0$ if $s<2 n-4$ so that Corollary (3.4) follows from Proposition (3.3) by the usual obstruction theory for fiber bundles. Since $\Pi_{2 n-4}\left(F_{n-1}, G_{n-1}\right)$ can be nonzero, Corollary (3.5) is proved in the same manner.

Corollary (3.6) follows directly from the homotopy sequence of the fibration (3.8) recalling that

$$
\begin{aligned}
\Pi_{s}\left(V_{n, 1}^{P L}\right) & =\Pi_{s}(P L(n) / P L(n, 1)) \cong \Pi_{s}(P L(n), P L(n, 1)) \\
& \cong \Pi_{s-1}(\Omega P L(n), \Omega P L(n, 1)) \cong \Pi_{s-1}\left(E\left(D^{1}, D^{1} \times \Sigma^{n-1} ; j\right)\right)
\end{aligned}
$$

and that $\Pi_{s}\left(\Sigma^{n-1}\right) \oplus \Pi_{s}\left(F_{n-1}, G_{n-1}\right) \cong \Pi_{s}\left(G_{n}, G_{n-1}\right)$.

4. Anomalous behavior near the stable range. The study of piecewise linear bundles is facilitated by a modification of an exact sequence of Kuiper and Lashof [17].

Proposition (4.1). There is an exact homotopy sequence

$$
\cdots \rightarrow \Pi_{i}\left(C_{n-1}\right) \rightarrow \Pi_{i}(P L(n-1)) \rightarrow \Pi_{i}(1 P L(n)) \rightarrow \cdots
$$

(4.3) Construction of the exact sequence. In the same manner that the quasifibration (2.28) was constructed via Proposition (2.14) in the proof of (2.27) one may construct a quasi-fibration

$$
H_{\partial_{0}}\left(D^{1} \backslash \Sigma^{n-1}, D^{1} \times \Sigma^{k-1}\right) \rightarrow H\left(D^{n}, D^{k}\right) \rightarrow G H\left(R^{n}, R^{k}\right)
$$

which, by taking $k=0$, becomes

$$
H_{\partial_{0}}\left(D^{1} \times \Sigma^{n-1}\right) \rightarrow H\left(D^{n},\{0\}\right) \stackrel{\gamma}{\longrightarrow} G H\left(R^{n},\{0\}\right) .
$$

A consequence of the Alexander Proposition (2.21) and the fibration

$$
H_{\partial}\left(D^{n},\{0\}\right) \rightarrow H\left(D^{n},\{0\}\right) \stackrel{\partial}{\longrightarrow} H\left(\Sigma^{n-1}\right)
$$


is that $\partial$ is a homotopy equivalence while Corollary (2.23)(iii) shows that $G H\left(R^{n},\{0\}\right)$ is homotopy equivalent to $H\left(R^{n},\{0\}\right)$. Up to homotopy $\gamma$ in (4.5) is equivalent to the inclusion $H\left(\Sigma^{n-1}\right) \subset H\left(R^{n},\{0\}\right)$ given by taking the open cone. Evaluation then gives a commutative diagram of fibrations:

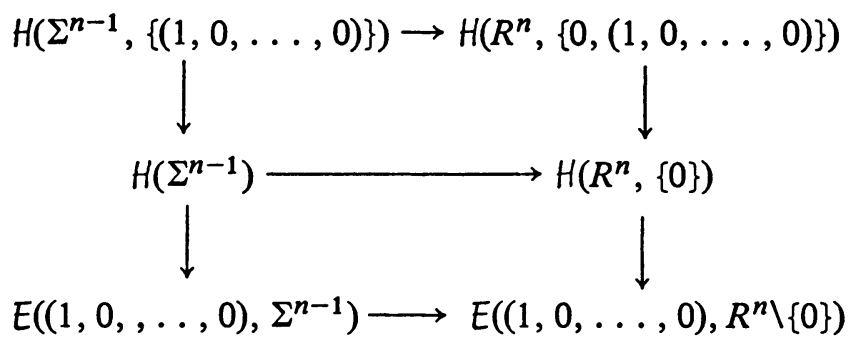

where the base spaces are homotopically equivalent (to $\Sigma^{n-1}$ ). One can identify $H\left(\Sigma^{n-1},\{(1,0, \ldots, 0)\}\right)$ as $P L(n-1)$ by noticing that the base space of the fibration

$$
\begin{aligned}
H\left(\Sigma^{n-1}, \Sigma^{0}\right) & \rightarrow H\left(\Sigma^{n-1},\{(1,0, \ldots, 0)\}\right) \\
& \rightarrow E\left((-1,0, \ldots, 0), \Sigma^{n-1} \backslash\{(1,0, \ldots, 0)\}\right)
\end{aligned}
$$

is contractible and applying (2.23), parts (ii) and (iii) with $m=0$. The inclusion of the fibers in (4.6) is just a disguised form of the inclusion of $P L(n-1)$ in $1 P L(n)$. The homotopy lattice of (4.6) then shows that $\Pi_{i}(1 P L(n), P L(n-1))$ is isomorphic to $\Pi_{i}\left(H\left(R^{n},\{0\}\right), H\left(\Sigma^{n-1}\right)\right)$. This is isomorphic to $\Pi_{i}$ (mapping cylinder $\left.\gamma, H\left(D^{n},\{0\}\right)\right)$ which, from (4.5), is isomorphic to $\Pi_{i-1}\left(H_{\partial_{0}}\left(D^{1} \times \Sigma^{n-1}\right)\right)$. If we let $C_{n-1}=C_{\partial_{0}}\left(\Sigma^{n-1}, \Sigma^{n-1} ; 1\right)=H_{\partial_{0}}\left(D^{1} \times \Sigma^{n-1}\right)$ and insert the isomorphism between $\Pi_{i}(1 P L(n), P L(n-1))$ and $\Pi_{i-1}\left(C_{n-1}\right)$ into the exact sequence of the pair (1PL(n), PL(n-1)) we have the sequence (4.2).

CoROllary (4.7). If $n \geqslant 4$, then $\iota_{*}: \Pi_{i}(P L(n-1)) \rightarrow \Pi_{i}(1 P L(n))$ is an isomorphism if $i \leqslant n$ and an epimorphism for $i=n+1$.

COROLlARY (4.8). The evaluation homomorphism

$$
\mathrm{ev}_{*}: \Pi_{i}(P L(n), P L(n-1)) \rightarrow \Pi_{i}\left(\Sigma^{n-1}\right)
$$

is an isomorphism for $i \leqslant n+1$ and an epimorphism for $i=n+2$.

(4.9) Proof of Corollaries (4.7) AND (4.8). In Theorem (5.3) of [20] it is shown that $\Pi_{i}\left(C_{\partial_{0}}\left(\Sigma^{n-1}, \Sigma^{n-1} ; 1\right)\right)$ is trivial for $i \leqslant n$. Thus Corollary (4.7) follows immediately from (4.2).

Recalling that in the proof of (4.1) it was shown that $\Pi_{i}\left(1 P L_{n}, P L_{n-1}\right) \cong$ $\Pi_{i-1}\left(C_{n-1}\right)$ Corollary (4.8) follows from the exact sequence of the triple $(P L(n), 1 P L(n), P L(n-1))$, 


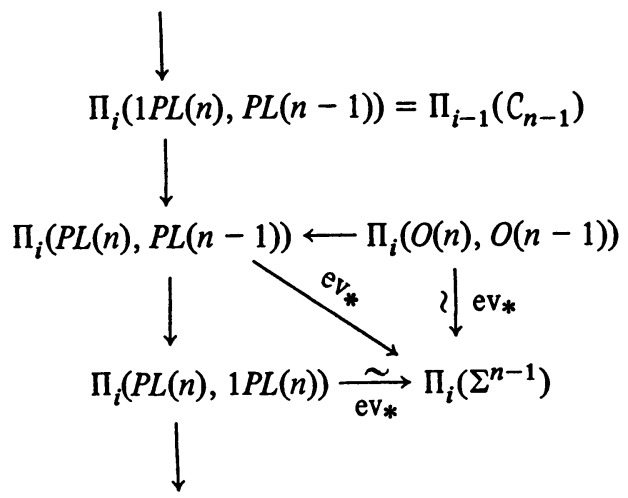

and Theorem (5.3) of [20].

(4.10) REMARK. Of course $\mathrm{ev}_{*}$ always gives a splitting of the exact sequence of the square $(P L(n) ; O(n), P L(n-1) ; O(n-1))=\square$ so that $\Pi_{i}(P L(n) / P L(n-1)) \cong$ $\Pi_{i}(O(n) / O(n-1)) \oplus \Pi_{i}(\square)$. In a personal communication R. Stern reminded me that Morlet [21] and Kirby and Siebenmann [16] have shown that for $n \geqslant 5$

$$
\Pi_{i+n+1}(\square) \cong \Pi_{i}\left(C_{\partial_{0}}^{\mathrm{DIFF}}\left(D^{n-1}, D^{n-1} ; 1 \mid \Sigma^{n-2}\right)\right)
$$

where the superscript DIFF denotes the extension of the definitions given in $\S 2$ to the differentiable category with the usual modifications near boundaries, $\mathrm{cf}$. [4], [21]. Cerf [8] has shown that $\Pi_{0}\left(C_{\partial_{0}}^{\mathrm{DIFF}}\left(D^{n-1}, D^{n-1} ; 1 \mid \Sigma^{n-2}\right)\right)=0$ if $n \geqslant$ 5 while Hatcher [11] and Volodin [26] have shown that

$$
\prod_{1}\left(C_{\partial_{0}}^{\mathrm{DIFFF}}\left(D^{n-1}, D^{n-1} ; 1 \mid \sum^{n-2}\right)\right) \cong \mathrm{Z}_{2}+w h_{3}(0)
$$

if $n \geqslant 9$. As a consequence one notes that $\Pi_{i}\left(C_{n-1}\right) \cong \Pi_{i+1}(\square)$, which is nontrivial for $i=n+1$.

Proposition (4.11). For $n \geqslant 9, \Pi_{n+2}(P L(n, 1), P L(n-1)) \neq 0$.

(4.12) Proof of (4.11). Proposition (3.3) states that $\Pi_{s}(1 P L(n), P L(n, 1)) \cong$ $\Pi_{s}\left(F_{n-1}, G_{n-1}\right)$ if $s<2 n-4$. As observed before, this latter group is trivial in this range so that $(4.2)$ implies that $\Pi_{n+2}(P L(n, 1), P L(n-1)) \cong \Pi_{n+1}\left(C_{n-1}\right)$. However, the previous remark shows that this group is nonzero.

(4.13) In summary we have shown in $\S 3$ that in the metastable range a nonzero section gives rise to a unique trivial sub- $R^{1}$-bundle, but that this fails just below the metastable range. The previous proposition shows that in the stable range a trivial sub- $R^{1}$-bundle splits the bundle but that this fails to hold just below the stable range. This failure of the splitting also shows that $\Pi_{n+2}(P L(n), \overline{P L}(n)) \neq 0$ if $n \geqslant 9$, cf. [25].

\section{REFERENCES}

1. T. Akiba, Homotopy types of some PL complexes, Bull. Amer. Math. Soc. 77 (1971), 1060-1062. MR 48 \#5090.

2. - Filling in cross sections of piecewise linear bundles, 1971 (mimeograph). 
3. J. W. Alexander, On the deformation of an n-cell, Proc. Nat. Acad. Sci. U.S.A. 9 (1923), 406-407.

4. P. L. Antonelli, D. Burghelea and P. J. Kahn, The concordance-homotopy groups of geometric automorphism groups, Lecture Notes in Math., vol. 215, Springer-Verlag, New York, 1971.

5. - The non-finite homotopy type of some diffeomorphism groups, Topology 11 (1972), 1-50.

6. M. G. Barratt, V. K. A. M. Gugenheim and J. C. Moore, On semisimplicial fibrebundles, Amer. J. Math. 81 (1959), 639-657. MR 22 \#1895.

7. W. Browder, Open and closed disk bundles, Ann. of Math. (2) 83 (1966), 218230. MR 32 \#6485.

8. J. Cerf, La stratification naturelle des espaces de fonctions différentiables réeles et le théorème de la pseudoisotopie, Inst. Hautes Études Sci. Publ. Math. No. 39 (1970), 5173. MR 45 \#1176.

9. E. B. Curtis, Simplicial homotopy theory, Advances in Math. 6 (1971), 107-209. MR 43 \#5529.

10. A. Haefliger and C. T. C. Wall, Piecewise linear bundles in the stable range, Topology 4 (1965), 209-214. MR 32 \#1716.

11. A. Hatcher, On the two-parameter h-cobordism theorem for two-connected manifolds, 1973 (mimeo).

12. J. F. P. Hudson, Concordance and isotopy of PL embeddings, Bull. Amer. Math. Soc. 72 (1966), 534-535. MR 33 \#1857.

13. - Piecewise linear topology, Benjamin, New York and Amsterdam, 1969. MR 40 \#2094.

14. Concordance, isotopy and diffeotopy, Ann. of Math. (2) 91 (1970), 425448. MR 41 \#4549.

15. I. M. James, On the iterated suspension, Quart. J. Math. Oxford Ser. (2) 5 (1954), 1-10. MR 15, 891 .

16. R. Kirby and L. Siebenmann, Classification of sliced families of smooth or piece. wise linear manifold structures.

17. N. H. Kuiper and R. K. Lashof, Microbundles and bundies. I, II, Invent. Math. 1 (1966), 1-17, 243-259. MR 35 \#7339; \#7340.

18. K. Millett, Piecewise linear concordance and isotopies, Mem. Amer. Math. Soc. No. $153,1974$.

19. K. Millett, Homotopy sequences of fibrations, Fund. Math. 79 (1973), 49-62. MR 48 \#5066.

20. - Homotopy groups of automorphism spaces, Geometric Topology, Lecture Notes in Math., vol. 438, Springer-Verlag, New York and Berlin, 1975.

21. C. Morlet, Plongements et automorphismes des variétés, Notes Cours Peccot, Collège de France, 1969.

22. C. P. Rourke and B. J. Sanderson, Block bundles. I, II, III, Ann. of Math. (2) 87 (1968), 1-28, 256-278, 431-483. MR 37 \#2234a, b; 38 \#729.

23. - An embedding without a normal microbundle, Invent. Math. 3 (1967), 293-299. MR 36 \#5954.

24. - $-\Delta$-sets I: Homotopy theory; $\Delta$-sets II: Blockbundles and block fibrations, Quart. J. Math. 22 (1971), 321-338. $257-269$.

25. R. Stern, On topological and piecewise linear vector fields, Topology 14 (1975),

26. I. A. Volodin, Algebraic K-theory, Uspehi Mat. Nauk 27 (1972), 207-208. (Russian)

27. E. C. Zeeman, Séminaire, Inst. Hautes Études Sci., 1962-1963 (mimeo). 